\title{
The Automatization of Alignment System for Calibration of Hydrometers
}

\begin{abstract}
G.S. SARIYERLi*, U.Y. AKCADAG And O. SAKARYA
The Scientific and Technical Research Council of Turkey, National Metrology Institute (TUBITAK-UME), Gebze, Turkey

(Received April 6, 2016; in final form November 23, 2016)

In this study, the determination of weighing liquid by using automatic alignment system and the reference density standard to realize the calibration of scale division by hydrostatic weighing method introduced by Cuckow in computer controll is planned. This system serves all projects of Volume, Density and Viscosity Laboratory for the calibration process of hydrometers and makes it fully automatic, fast, more precise and efficient in comparison with manual calibration system. A new method refers automatically specific scale-marks for the calibration of hydrometers. A hydrometer calibration system adopting the new method consists of a vision system, positioning frame by servo motor and software to control the system. The vision system is composed of a camera to acquire images of meniscus level conflicted with scale level of hydrometer. The position of temperature controlled bath is provided by the positioning frame controlled with PLC. The stepping motor moves the camera, which is attached to the vessel containing a reference liquid, along the hydrometer. The aim of this study is to represent and validate the automated hydrometer calibration system of TUBITAK UME realizing the hydrometer comparison $\left(1000-1020 \mathrm{~kg} / \mathrm{m}^{3}\right)$ between automated and manual hydrometer calibration system.
\end{abstract}

DOI: 10.12693/APhysPolA.131.1466

PACS/topics: the Cuckow method, hydrostatic weighing, hydrometer, density, calibration, meniscus

\section{Introduction}

Hydrometers, also known as gravimeters, areometers or densimeters, are the most ancient, simple and highly effective instruments, widely used at different levels of accuracy, to measure the density of liquids from $0.5 \mathrm{~g} / \mathrm{cm}^{3}$ to $2.0 \mathrm{~g} / \mathrm{cm}^{3}[1,2]$.

Hydrometers can be used to measure any liquid property that is a function of density simply by putting an appropriate scale in the hydrometer stem (Fig. 1).

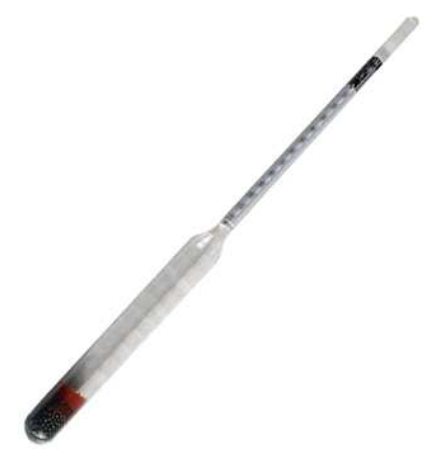

Fig. 1. Density hydrometer.

There are two primary methods for calibrating hydrometers. First one requires floating the hydrometer in several liquids of different densities, which are determined

\footnotetext{
* corresponding author; e-mail: gokce.sariyerli@tubitak.gov.tr
}



Fig. 2. Hydrostatic reading in liquids.

by applying the Archimedes principle to a sinker of known mass and volume (Fig. 2). The second method (the Cuckow method) requires hydrostatic weighing, i.e. using a balance to measure the mass of the hydrometer while it is immersed to several levels in an unique liquid of known density.

The Cuckow method has many advantages. It eliminates the inconvenience of storing a collection of liquids. It provides the calibration of a hydrometer at specified scale marks without the inconvenience of preparing liquid mixtures to specified densities. This method can be automated more easily.

Volume, Density and Viscosity laboratory of UME is willing to automate the calibration of hydrometers in order to reduce uncertainties and provide more accurate 
measurements. It was built an automated and primary standard system for the calibration of hydrometers using the Cuckow method [3].

The primary level hydrometer calibration system of volume, density and viscosity laboratory immerses the hydrometer chosen for test far enough into tridecane liquid so that the hydrometer mark to be calibrated is aligned with the free surface.

\section{Method and theoretical equations}

The method is based on weighing of the hydrometer when it has been floating at the scale mark, which is supposed to be calibrated. Density of the liquid must be previously measured. Hydrometer experiences an upthrust equal to its weight when it is immersed up to the scale mark and order of upthrust is proportional to the amount of immersion. If we weigh the body immersed to requested extent in two liquids of known density it would be possible to determine the density corresponding to requested extent which is supposed to be a scale mark. Application of this procedure requires special apparatus and special designs for sensitive immersion $[3,4]$.

Theory of the method is based on the fact that, if we weigh a solid body at a point in between two media whose densities are known, we may find the density of the medium in which the upthrust on the immersed portion would be equal to the weight of the body. Hydrometers are firstly weighed in air and then in a reference liquid of known density, which is used as reference. The weighing value is measured while the hydrometers were immersed up to different levels.

The density corresponds related scale line can be determined with the equation by using the measurement results according to the Cuckow method at reference temperature $\left(20^{\circ} \mathrm{C}\right.$ or $\left.15^{\circ} \mathrm{C}\right)$.

$$
\begin{aligned}
& \rho\left(t_{B}\right)=\left[\rho_{F}(t)-\rho_{L L}\right] \frac{W_{L}+(\pi / g) d \gamma\left(t_{B}\right)}{W_{L}-W_{F}+(\pi / g) d \gamma_{F}(t)} \\
& \quad \times\left[1+\alpha_{V}\left(t-t_{B}\right)\right]+\rho_{L L} .
\end{aligned}
$$

In Eq. (1): $\rho\left(t_{B}\right)$ - density of calibrated scale line, $W_{L}$ - weighing value of hydrometer in air, $W_{F}$ - weighing value of hydrometer in liquid, $t_{B}$ - reference temperature, $t$ - temperature during measurement, $d$ - stem diameter of hydrometer at meniscus level, $\rho_{F}(t)-$ density of liquid during measurement, $\rho_{L L}$ - density of air during weighing in air, $\gamma\left(t_{B}\right)$ - surface tension of reference liquid, $\gamma_{F}(t)$ - surface tension of tridecane, $\alpha_{V}$ hydrometer's volumetric expansion coefficient, $g$ - local gravitational acceleration.

The air density can be determined by Eq. (2):

$$
\rho_{a}=\frac{0.34844 P-h(0.00252 t-0.020582)}{273.15+t} .
$$

The liquid density $\left(\rho_{F}(t)\right)$ shall be determined by sinker (reference density standard) using substitute method with the same system in air and liquid media $[4,5]$. In Eq. (2): $\rho_{a}$ - air density $\left[\mathrm{kg} / \mathrm{m}^{3}\right], t-$ environmental temperature $\left[{ }^{\circ} \mathrm{C}\right], P-$ atmospheric pressure $[\mathrm{mbar} / \mathrm{hPa}], h$ - environmental humidity [\% rh].

\section{Automated hydrometer calibration system}

A manual hydrometer calibration system had been used in Volume, Density and Viscosity Laboratory shown in Fig. 3. The hydrometer shall be immersed into the calibration liquid up to the calibration scale mark by the hydrostatic weighing method for calibration process. The hydrometer is bounded up with balance by holder and hook assembly. The calibration liquid takes place in the glass tube positioned into the temperature controlled bath [6].

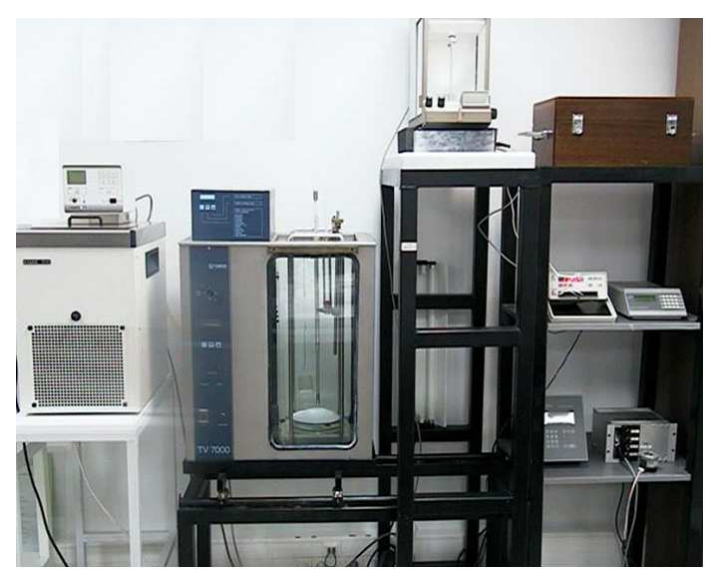

Fig. 3. A manual hydrometer calibration system.

The hydrometer calibration system described here consists of an electronic balance, a frame, a glass vessel containing a reference liquid, a thermometer, a hygrometer, a barometer, and an automatic alignment system. A cross section view of automated hydrometer system can be seen in Fig. 4.



Fig. 4. Automated hydrometer calibration system.

The software comprises a camera image to search for a meniscus and target scale-marks, and a program to control the stepping motor that aligns the temperature controlled bath on frame grabber (Fig. 5). It can be seen the position of meniscus before and after adjustment using alignment system by hand control unit (Fig. 6). 


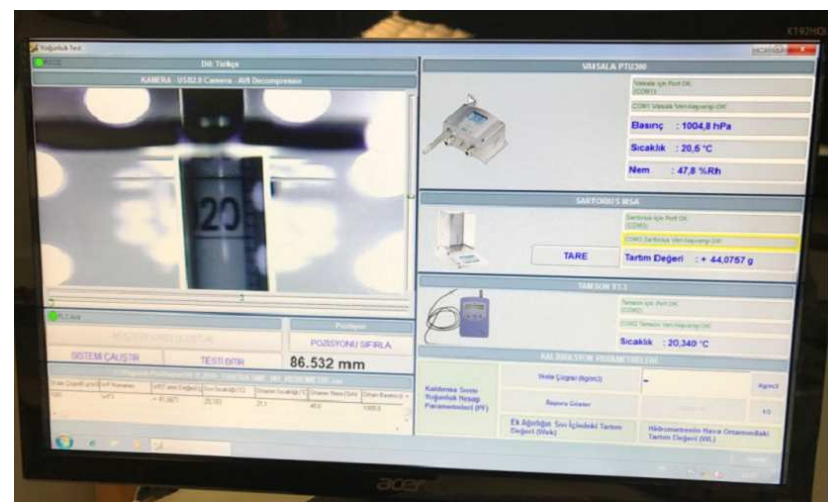

Fig. 5. A camera image (calibration point $1019 \mathrm{~kg} / \mathrm{m}^{3}$ ) of immersed part of the hydrometer under test.
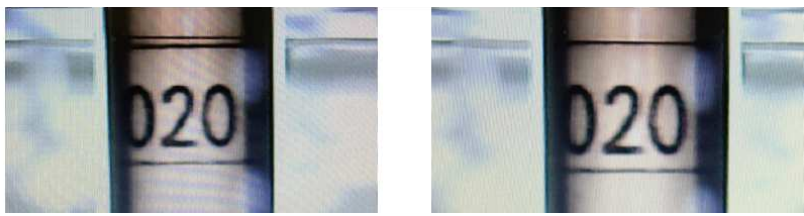

Fig. 6. A camera image before and after meniscus adjustment.

Figure 7 shows the configuration of the automated hydrometer calibration system. The automatic alignment system consists of a camera, a frame grabber, a light, a stepping motor, and software to control the system. The distance between the hydrometer and the camera is $150 \mathrm{~mm}$. The glass vessel is cylindrical with a height

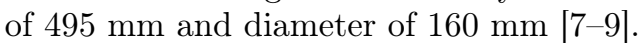

\section{Experimental setup}

Firstly measurements are carried out in air medium with a balance having $0.1 \mathrm{mg}$ readability.

Calibrated weights are used in substitution method and air density is calculated by using ambient conditions so that air buoyancy correction would be concerned in weighing value. Right after weighing hydrometers in air, it is important to measure stem diameters at required scale marks of hydrometer, and reference surface tensions of the liquid, where the hydrometer is supposed to be used, are determined from related tables. Surface tension of the calibration liquid (tridecane) is already known. Second stage is to perform hydrostatic "Cuckow" weighing. In this process the balance is located at the top of the system and it has the ability to perform measurements inside the liquid via an apparatus, which connects the balance into the liquid. Temperature controlled bath is used for temperature stabilization. Actually, temperature is the major effect for the density of liquid in which the weighing takes place. Ambient conditions are monitored and recorded by $\mathrm{PC}$ during the weighing in liquid.

The calibration process of hydrometer is defined as follows. Heated controlled bath should be adjusted before the calibration process. A system should reach a thermal equilibrium with using heated controlled bath and cooler. Next an adjusted hydrometer holder hanging on balance in air ambient is tared and environmental conditions (temperature, atmospheric pressure, and humidity) are recorded. The hydrometer is tightened into the holder and then it is immersed in a liquid until a scale line overlaps with a liquid level. The liquid level can be displaced with the immersion of displacement object and the overlapping (reading can be realized with magnificence) of scale line and liquid level are provided (if the calibration of scale line of lower density hydrometer is realized, known mass of stainless steel is used as an additional weight) (Fig. 8). After that weight value and temperature of liquid can be measured. The measurements are realized in 5 different scale lines (the scale lines can be adjusted by holder). The determination of density value of hydrometer scale lines are realized by using the measurement results as part of the Cuckow method. The density values of calibrated hydrometer are given in calibration certificate at reference temperature $\left(15^{\circ} \mathrm{C}, 20^{\circ} \mathrm{C}\right.$ or a request of customer).

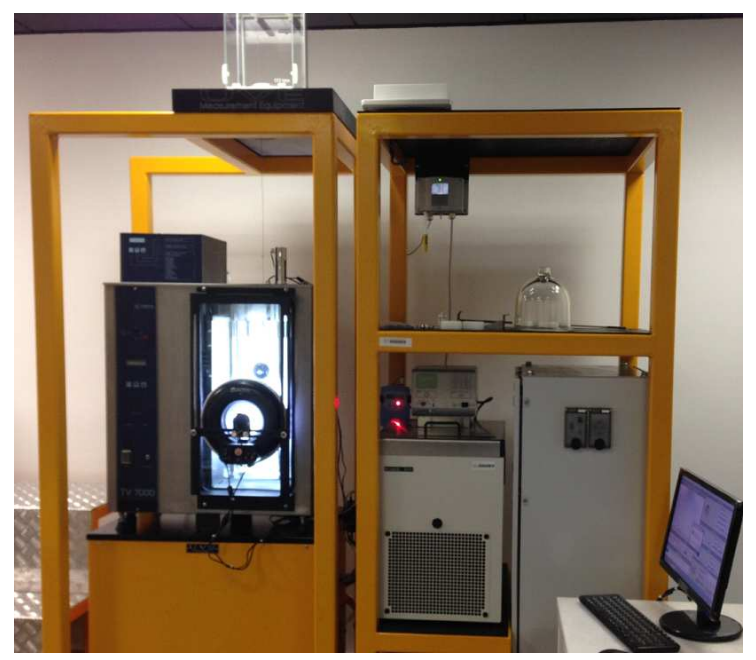

Fig. 7. A cross-section view of automated hydrometer system.

\section{Measurement and results}

Experiments were performed to show the accuracy and sensitivity of automated calibration system. A hydrometer with a range of $1000 \mathrm{~kg} / \mathrm{m}^{3}$ to $1020 \mathrm{~kg} / \mathrm{m}^{3}$ was used in the measurements.

The standard hydrometer was selected to compare the results of both automated and manual calibration system with the comparison range cover different densities of hydrometer.

The obtained results are given in Table I. All the calibration points, error, expanded uncertainty and $E_{n}$ values between automated and manual calibration system. The automated calibration system was taken as a reference in all measurements.

The formula seen below for the normalized error ( $E_{n}$-value) was used. 


$$
E_{n}=\left|\frac{\text { Error }_{\text {manual }}-\text { Error }_{\text {automated }}}{\sqrt{\mathrm{U}_{\text {manual }}^{2}+\mathrm{U}_{\text {automated }}^{2}}}\right| \leq 1 .
$$

Error $_{\text {manual }}=$ Measured value $_{\text {manual }}$ Nominal value manual, Error $_{\text {automated }}$ = Measured value automated - Nominal value automated $_{\text {, }}$ Error $_{\text {manual }}$ is the value reported by the manual hydrometer calibration system, Error automated is the reference value by the automated calibration system, $U_{\text {manual }}$ is the uncertainty of the manual hydrometer calibration system, $U_{\text {automated }}$ is the uncertainty of the automated hydrometer calibration system.

TABLE I

The results of measurements.

\begin{tabular}{c|c|c|c|c|c}
\hline \hline \multicolumn{2}{c}{ Range of hydrometer $\left[\mathrm{kg} / \mathrm{m}^{3}\right]$} & \multicolumn{3}{|c}{$1000-1020$} \\
\hline \multirow{2}{*}{ nominal } & $\begin{array}{c}\text { automated hydrometer } \\
\text { calibration system }\end{array}$ & \multicolumn{2}{|c}{$\begin{array}{c}\text { manual hydrometer } \\
\text { calibration system }\end{array}$} & \multirow{2}{*}{$E_{n}$} \\
\cline { 2 - 5 } value & error & $\begin{array}{c}\text { expanded } \\
\text { uncertainty }\end{array}$ & error & $\begin{array}{c}\text { expanded } \\
\text { uncertainty }\end{array}$ & value \\
{$\left[\mathrm{kg} / \mathrm{m}^{3}\right]$} & {$\left[\mathrm{kg} / \mathrm{m}^{3}\right]$} & {$\left[\mathrm{kg} / \mathrm{m}^{3}\right]$} & {$\left[\mathrm{kg} / \mathrm{m}^{3}\right]$} & {$\left[\mathrm{kg} / \mathrm{m}^{3}\right]$} & \\
\hline 1001 & -0.657 & 0.025 & -0.641 & 0.139 & 0.11 \\
1004 & -0.624 & 0.025 & -0.654 & 0.144 & 0.21 \\
1015 & -0.632 & 0.025 & -0.626 & 0.141 & 0.04 \\
1019 & -0.585 & 0.025 & -0.649 & 0.141 & 0.44
\end{tabular}

Measurement results are evaluated as the definition below.

The result in the range $-1 \leq E_{n} \leq 1$ is satisfactory and the result $E_{n}>1$ or $E_{n}<-1$ is unsatisfactory.

In measurement processes, the hydrometer used in the experiment was calibrated while the reference liquid (tridecane) was kept at $20^{\circ} \mathrm{C}$ during measurements. It was provided to keep the density of tridecane constant. In both the manual and automatic calibrations, the same scale-marks were used. The comparison results of two systems can be seen in Fig. 8 .



Fig. 8. The comparison results of automated and manual calibration system.

\section{Conclusion}

In this paper, there was described an automatic system for calibrating a hydrometer. The new system reduces errors caused by operator skills or attention when process is performed manually. The new calibration system automatically aligns specific hydrometer scale-marks with the horizontal plane of the reference liquid. A camera, a frame grabber, a step motor, and a software program are used to realize this process. A linear-motion controller is added to the hydrometer calibration equipment, and approaches the desired position slowly in order not to disturb the horizontal plane of the reference liquid.

In this study the meniscus is matched with reference liquid line to focus on accurate calibration point.

A new procedure is realized to offer calibration services of reference standard hydrometers to their customers more accurate and with less uncertainty of measurement by Volume, Density and Viscosity Laboratory of TUBITAK UME.

The automated hydrometer calibration system was designed, constructed and validated for using the system in hydrometer calibrations in the range between $600 \mathrm{~kg} / \mathrm{m}^{3}$ to $2000 \mathrm{~kg} / \mathrm{m}^{3}$.

The evaluation of expanded uncertainty of both system is mainly affected by the individual contributions of the density of the reference liquid, the temperature of calibration liquid and the reading of the meniscus. The uncertainty value of hydrometer calibration with manual system is decreased with the ratio of $1 / 4$ compared with automated system due to the improved parameters.

\section{References}

[1] ISO 649-1: 1981, "Laboratory Glassware-Density Hydrometers for General Purposes, Part-1 Specification".

[2] ISO 649-2: 1981, "Laboratory Glassware-Density Hydrometers for General Purposes, Part-2 Test Methods and Use".

[3] F.W.Cuckow, J. Soc. Chem. Industry 68, 44 (1949).

[3] F.W. Cuckow, J. Soc. Chem. Industry 68, 44 (1949).

[4] NIST Special Publication 250-78, "NIST Calibration Services for Hydrometers", January 14, 2008.

[5] S.Lorefice, A. Malengo, Meas. Sci. Technol. 17, 2560 (2006).

[6] U.Y. Akcadag, S.E. San, in: Proc. 17th Int. Conf. on Force, Mass, Torque and Pressure Measurements, IMEKO TC3, Istanbul (Turkey), Curran Associates, Red Hook (NY) 2001.

[7] Y.J. Lee, K.H. Chang, J.C. Chon, C.Y. Oh, Metrologia 41, S100 (2004).

[8] L.M. Peńa, J.C. Pedraza, L.O. Becerra, C.A. Galvan, in: IMEKO 20th TC3, 3rd TC16 and 1st TC22 Int. Conf. Cultivating Metrological Knowledge, Merida (Mexico), 2007.

[9] L.M. Peńa-Perez, J.C. Pedraza-Ortega, J.M. RamosArreguin, S. Tovar-Arriaga, M.A. Aceves-Fernandez, L.O. Becerra, E. Gorrostieta-Hurtado, J.E. VargasSoto, Sensors 13, 14367 (2013). 